\title{
First search for neutrinos in correlation with gamma-ray bursts with the ANTARES neutrino telescope
}

S. Adrián-Martínez ${ }^{a}$ I. Al Samarai ${ }^{b}$ A. Albert $^{c}$ M. André ${ }^{d} M$. Anghinolfi ${ }^{e}$ G. Anton ${ }^{f}$ S. Anvar ${ }^{g}$ M. Ardid ${ }^{a}$ A.C. Assis Jesus ${ }^{h}$ T. Astraatmadja, ${ }^{h, 1}$ J-J. Aubert ${ }^{b}$ B. Baret ${ }^{i}$ S. Basa ${ }^{j}$ V. Bertin ${ }^{b}$ S. Biagi $^{k, l}$ C. Bigongiari ${ }^{n}$ C. Bogazzi ${ }^{h}$ M. Bou-Cabo ${ }^{a}$ B. Bouhou ${ }^{i}$ M.C. Bouwhuis, ${ }^{h, 2}$ J. Brunner ${ }^{b}$ J. Busto ${ }^{b}$ A. Capone ${ }^{o, p}$ C. Cârloganu ${ }^{q}$ J. Carr $^{b}$ S. Cecchini ${ }^{k}$ Z. Charif ${ }^{b} \mathbf{P h}$. Charvis ${ }^{r}$ T. Chiarusi $^{k}$ M. Circella ${ }^{s}$ R. Coniglione ${ }^{t}$ L. Core ${ }^{b}$ H. Costantini ${ }^{b}$ P. Coyle $^{b}$ A. Creusot ${ }^{i}$ C. Curtil ${ }^{b}$ G. De Bonis ${ }^{o, p}$ M.P. Decowski ${ }^{h}$ I. Dekeyser $^{u}$ A. Deschamps $r$ C. Distefano ${ }^{t}$ C. Donzaud ${ }^{i, v}$ D. Dornic ${ }^{b}$ Q. Dorosti ${ }^{w}$ D. Drouhin ${ }^{c}$ T. Eberl ${ }^{f}$ U. Emanuele ${ }^{n}$ A. Enzenhöfer ${ }^{f}$ J-P. Ernenwein ${ }^{b}$ S. Escoffier ${ }^{b}$ K. Fehn ${ }^{f}$ P. Fermani ${ }^{o, p}$ M. Ferri ${ }^{a}$ S.

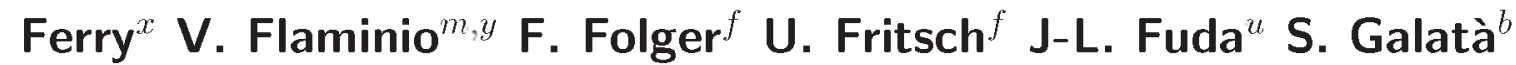
P. Gay ${ }^{q}$ K. Geyer ${ }^{f}$ G. Giacomellik ${ }^{k, l}$ V. Giordano ${ }^{t}$ J.P.

Gómez-González $^{n}$ K. Graf ${ }^{f}$ G. Guillard ${ }^{q}$ G. Hallewell ${ }^{b}$ M. Hamal ${ }^{a l}$ H. van Haren ${ }^{z}$ A.J. Heijboer ${ }^{h}$ Y. Hello ${ }^{r}$ J.J. Hernández-Rey ${ }^{n}$ B.

${ }^{1}$ Also at University of Leiden, the Netherlands

${ }^{2}$ Corresponding author, Email address: mieke.bouwhuis@nikhef.nl 
Herold $^{f}$ J. Hößl ${ }^{f}$ C.C. Hsu ${ }^{h}$ M. de Jong ${ }^{h, 1}$ M. Kadler ${ }^{a a}$ O. Kalekin ${ }^{f}$ A. Kappes, ${ }^{f, 3}$ U. Katz ${ }^{f}$ O. Kavatsyuk ${ }^{w}$ P. Kooijman ${ }^{h, a b, a c}$ C. Kopper $^{h, f}$ A. Kouchner ${ }^{i}$ I. Kreykenbohm ${ }^{a a}$ V. Kulikovskiy ${ }^{a d, e}$ R. Lahmann $^{f}$ G. Lambard ${ }^{n}$ G. Larosa ${ }^{a}$ D. Lattuada ${ }^{t}$ D. Lefèvre ${ }^{u}$ G.

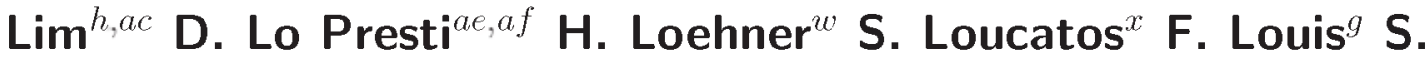
Mangano $^{n}$ M. Marcelin ${ }^{j}$ A. Margiotta ${ }^{k, l}$ J.A. Martínez-Mora ${ }^{a}$ T. Montaruli $^{s, a g}$ M. Morganti, ${ }^{m, 4}$ L. Moscoso, ${ }^{i, x, 5}$ H. Motz ${ }^{f}$ M. Neff ${ }^{f}$ E. Nezri ${ }^{j}$ D. Palioselitis ${ }^{h}$ G.E. Păvălaș ${ }^{a h}$ K. Payet ${ }^{x}$ J. Petrovic ${ }^{h}$ P. Piattellit $^{t}$ V. Popa ${ }^{a h}$ T. Pradier ${ }^{a i}$ E. Presani ${ }^{h}$ C. $\operatorname{Racca}^{c}$ C. Reed ${ }^{h}$ G. Riccobene $^{t}$ C. Richardt ${ }^{f}$ R. Richter ${ }^{f}$ C. Rivière ${ }^{b} \mathbf{A}$. Robert ${ }^{u}$ K. Roensch $^{f}$ A. Rostovtsev ${ }^{a j}$ J. Ruiz-Rivas ${ }^{n}$ M. Rujoiu ${ }^{a h}$ G.V. Russo $^{a e, a f}$ F. Salesa ${ }^{n}$ D.F.E. Samtleben ${ }^{h}$ A. Sánchez-Losa ${ }^{n}$ P. Sapienza $^{t}$ J. Schnabel ${ }^{f}$ F. Schöck ${ }^{f}$ J-P. Schuller ${ }^{x}$ F. Schüssler ${ }^{x}$ T. Seitz ${ }^{f}$ R. Shanidze ${ }^{f}$ F. Simeone ${ }^{o, p}$ A. Spies ${ }^{f}$ M. Spurio ${ }^{k, l}$ J.J.M. Steijger $^{h}$ Th. Stolarczyk ${ }^{x}$ M. Taiuti ${ }^{e, a k}$ C. Tamburini ${ }^{u}$ A. Trovato ${ }^{a e}$ B. Vallage ${ }^{x}$ C. Vallée ${ }^{b}$ V. Van Elewyck ${ }^{i}$ M. Vecchi ${ }^{b}$ P. Vernin ${ }^{x}$ E. Visser $^{h}$ S. Wagner ${ }^{f}$ G. Wijnker ${ }^{h}$ J. Wilms ${ }^{a a}$ E. de Wolf ${ }^{h, a c} \mathbf{H}$. Yepes $^{n}$ D. Zaborov ${ }^{a j}$ J.D. Zornoza ${ }^{n}$ J. Zúñiga ${ }^{n}$

\footnotetext{
${ }^{a}$ Institut d'Investigació per a la Gestió Integrada de les Zones Costaneres (IGIC) - Universitat Politècnica de València.

C/ Paranimf 1, 46730 Gandia, Spain.

${ }^{b}$ CPPM, Aix-Marseille Université, CNRS/IN2P3, Marseille, France

${ }^{c}$ GRPHE - Institut universitaire de technologie de Colmar, 34 rue du Grillenbreit BP 50568 - 68008 Colmar, France

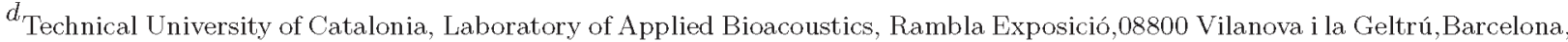
Spain

${ }^{e}$ INFN - Sezione di Genova, Via Dodecaneso 33, 16146 Genova, Italy

${ }^{f}$ Friedrich-Alexander-Universität Erlangen-Nürnberg, Erlangen Centre for Astroparticle Physics, Erwin-Rommel-Str. 1, 91058 Erlangen, Germany

${ }^{g}$ Direction des Sciences de la Matière - Institut de recherche sur les lois fondamentales de l'Univers - Service d'Electronique des Détecteurs et d'Informatique, CEA Saclay, 91191 Gif-sur-Yvette Cedex, France

${ }^{h}$ Nikhef, Science Park, Amsterdam, The Netherlands

${ }^{i}$ APC - Laboratoire AstroParticule et Cosmologie, UMR 7164 (CNRS, Université Paris 7 Diderot, CEA, Observatoire de Paris) 10, rue Alice Domon et Léonie Duquet 75205 Paris Cedex 13, France

${ }^{j}$ LAM - Laboratoire d'Astrophysique de Marseille, Pôle de l'Étoile Site de Château-Gombert, rue Frédéric Joliot-Curie 38, 13388 Marseille Cedex 13, France

${ }^{k}$ INFN - Sezione di Bologna, Viale Berti-Pichat 6/2, 40127 Bologna, Italy

${ }^{l}$ Dipartimento di Fisica dell'Università, Viale Berti Pichat 6/2, 40127 Bologna, Italy

${ }^{m}$ INFN - Sezione di Pisa, Largo B. Pontecorvo 3, 56127 Pisa, Italy

${ }^{n}$ IFIC - Instituto de Física Corpuscular, Edificios Investigación de Paterna, CSIC - Universitat de València, Apdo. de Correos 22085, 46071 Valencia, Spain
}

\footnotetext{
${ }^{3}$ On leave of absence at Humboldt-Universität zu Berlin

${ }^{4}$ Also at Accademia Navale de Livorno, Livorno, Italy

${ }^{5}$ Deceased
} 
${ }^{o}$ INFN -Sezione di Roma, P.le Aldo Moro 2, 00185 Roma, Italy

${ }^{p}$ Dipartimento di Fisica dell'Università La Sapienza, P.le Aldo Moro 2, 00185 Roma, Italy

${ }^{q}$ Clermont Université, Université Blaise Pascal, CNRS/IN2P3, Laboratoire de Physique Corpusculaire, BP 10448, 63000 Clermont-Ferrand, France

${ }^{r}$ Géoazur - Université de Nice Sophia-Antipolis, CNRS/INSU, IRD, Observatoire de la Côte d'Azur and Université Pierre et Marie Curie, BP 48, 06235 Villefranche-sur-mer, France

${ }^{s}$ INFN - Sezione di Bari, Via E. Orabona 4, 70126 Bari, Italy

${ }^{t}$ INFN - Laboratori Nazionali del Sud (LNS), Via S. Sofia 62, 95123 Catania, Italy

${ }^{u}$ COM - Centre d'Océanologie de Marseille, CNRS/INSU et Université de la Méditerranée, 163 Avenue de Luminy, Case 901, 13288 Marseille Cedex 9, France

${ }^{v}$ Univ Paris-Sud, 91405 Orsay Cedex, France

${ }^{w}$ Kernfysisch Versneller Instituut (KVI), University of Groningen, Zernikelaan 25, 9747 AA Groningen, The Netherlands

${ }^{x}$ Direction des Sciences de la Matière - Institut de recherche sur les lois fondamentales de l'Univers - Service de Physique des Particules, CEA Saclay, 91191 Gif-sur-Yvette Cedex, France

${ }^{y}$ Dipartimento di Fisica dell'Università, Largo B. Pontecorvo 3, 56127 Pisa, Italy

${ }^{z}$ Royal Netherlands Institute for Sea Research (NIOZ), Landsdiep 4,1797 SZ 't Horntje (Texel), The Netherlands

${ }^{a a}$ Dr. Remeis-Sternwarte and ECAP, Universität Erlangen-Nürnberg, Sternwartstr. 7, 96049 Bamberg, Germany

${ }^{a b}$ Universiteit Utrecht, Faculteit Betawetenschappen, Princetonplein 5, 3584 CC Utrecht, The Netherlands

${ }^{a c}$ Universiteit van Amsterdam, Instituut voor Hoge-Energie Fysika, Science Park 105, 1098 XG Amsterdam, The Netherlands

${ }^{a d}$ Moscow State University,Skobeltsyn Institute of Nuclear Physics, Leninskie gory, 119991 Moscow, Russia

${ }^{a e}$ INFN - Sezione di Catania, Viale Andrea Doria 6, 95125 Catania, Italy

${ }^{a f}$ Dipartimento di Fisica ed Astronomia dell'Università, Viale Andrea Doria 6, 95125 Catania, Italy

${ }^{a g}$ Département de Physique Nucléaire et Corpusculaire, Université de Genève, 1211, Geneva, Switzerland

${ }^{a h}$ Institute for Space Sciences, R-77125 Bucharest, Măgurele, Romania

${ }^{a i}$ IPHC-Institut Pluridisciplinaire Hubert Curien - Université de Strasbourg et CNRS/IN2P3 23 rue du Loess, BP 28, 67037 Strasbourg Cedex 2, France

${ }^{a j}$ ITEP - Institute for Theoretical and Experimental Physics, B. Cheremushkinskaya 25, 117218 Moscow, Russia

${ }^{a k}$ Dipartimento di Fisica dell'Università, Via Dodecaneso 33, 16146 Genova, Italy

${ }^{a l}$ University Mohammed I, Laboratory of Physics of Matter and Radiations, B.P.717, Oujda 6000, Morocco

Abstract. A search for neutrino-induced muons in correlation with a selection of 40 gammaray bursts that occurred in 2007 has been performed with the ANTARES neutrino telescope. During that period, the detector consisted of 5 detection lines. The ANTARES neutrino telescope is sensitive to $\mathrm{TeV}-\mathrm{PeV}$ neutrinos that are predicted from gamma-ray bursts. No events were found in correlation with the prompt photon emission of the gamma-ray bursts and upper limits have been placed on the flux and fluence of neutrinos for different models. 


\section{Introduction}

Gamma-ray bursts (GRBs), transient flashes of gamma-rays having a duration of sub-seconds up to several hundred seconds, are the most powerful known extra-galactic events. Several models predict a burst of high-energy neutrinos in concurrence with the flash of gamma-rays, also referred to as the prompt emission (see e.g. [1] for a review). In this, neutrinos are produced by interactions of protons that are accelerated by shock waves with energetic ambient photons. A variety of models has been put forth covering a wide range in the expected amount of neutrinos. Some of those models have already been challenged by the recent IceCube measurements [2]. In particular, the IceCube results do not support the notion of GRBs as primary source of ultra high-energy cosmic rays, at least under the assumption that these ultra high-energy cosmic rays are protons coming from the decay of photohadronically produced neutrons (i.e. via the resonant channel $p+\gamma \rightarrow \Delta^{+} \rightarrow n+\pi^{+}$) and are therefore connected to neutrinos. In this scenario, neutrons, as opposed to the protons, can escape because they are not magnetically confined inside the source. Nevertheless, a viable phase space of models with a potentially measurable neutrino flux from GRBs in the TeV-PeV region can still be tested for a better understanding of the nature of these energetic events.

In this paper, the first data taken with the ANTARES neutrino telescope in 2007 are used to perform a search for $\mathrm{TeV}-\mathrm{PeV}$ neutrinos in correlation with a selection of GRBs detected by satellite observatories. The data are treated in a stacking approach in which the data observed during the prompt emission of all the selected GRBs are accumulated. The detection of a single neutrino event would constitute an observation with more than $3 \sigma$ significance.

Previous searches for neutrinos from GRBs have been performed in the Northern hemisphere by AMANDA [3] and IceCube [4, 5] at similar energies ( $\mathrm{TeV}-\mathrm{PeV}$ ) and by ANITA [6] at higher energies (>PeV). IceCube also included some GRBs in the Southern hemisphere [2] that is observed by ANTARES but not during the period considered in this analysis. Other searches in the Southern hemisphere were performed by Super-Kamiokande [7] at lower energies $(\mathrm{MeV}-100 \mathrm{TeV})$ and by the Baikal neutrino telescope NT200 [8]. Both these experiments have a lower sensitivity than the current analysis in the comparable energy range. The ANTARES neutrino telescope is the most sensitive instrument to observe high-energy neutrinos from the GRBs considered in this analysis. In addition, the vast majority of these GRBs have not been studied for neutrino emission in the $\mathrm{TeV}-\mathrm{PeV}$ range before. Despite the fact that more stringent limits have been published $[2,4,5]$ for different GRB samples, ANTARES might still have observed events from the GRB sample presented here.

The completed ANTARES detector is the largest neutrino telescope on the Northern hemisphere and is sensitive to neutrinos in the $\mathrm{TeV}$ to $\mathrm{PeV}$ energy range. Located in the Mediterranean Sea, the ANTARES detector is sensitive to GRBs in the Southern hemisphere where the sensitivity of IceCube significantly suffers from the large background from muons produced by cosmic ray interactions in the atmosphere above the detector. Due to the transient nature of the GRBs and the variety of their characteristics, it is essential to permanently monitor the full sky in order to maximise the probability to observe a neutrino signal. The search described in this paper is the first in a series of searches which will be pursued with the data that will be acquired by the completed ANTARES detector up to 2016 and then continued with data from the future KM3NeT [9] neutrino observatory in the Mediterranean Sea. 


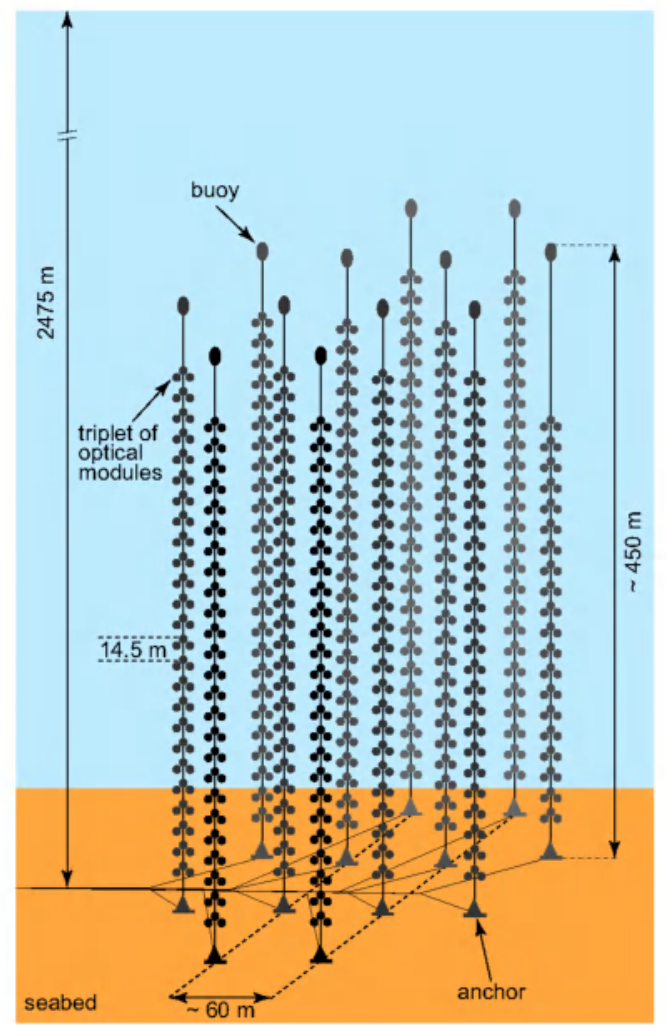

Figure 1. Schematic view of the ANTARES detector in its final configuration.

\section{Neutrino detection}

High-energy neutrinos can be detected indirectly by a neutrino telescope such as ANTARES. The detection principle relies on measuring the Cherenkov light induced by high-energy charged particles that are produced in a neutrino interaction inside or near the instrumented volume. In particular, a high-energy muon is produced in a charged current muon-neutrino interaction. High-energy muons can travel large distances, which facilitates an accurate determination of the direction. At the typical neutrino energies considered in this analysis ( $5 \mathrm{TeV}-6 \mathrm{PeV}$ ), the direction of the muon closely follows that of the incident neutrino. Above $5 \mathrm{TeV}$ the median angle between the neutrino direction and the muon direction is less than $0.3^{\circ}$. The direction of a high-energy muon can thus be correlated to the positions of GRBs.

The Cherenkov light is detected by photo-multiplier tubes, housed in optical modules [10]. Triplets of optical modules are attached to vertical detection lines of about 450 metres height, which are anchored to the sea bed at a depth of 2475 metres and held upright by a buoy. In its final configuration, the ANTARES detector consists of 12 detection lines with a spacing of about 60 metres (see figure 1). Eleven of these detection lines each contain 25 evenly spaced triplets of optical modules and one contains 20 triplets. The positions and orientations of the optical modules vary due to the sea currents. An acoustic positioning system, combined with compasses and tiltmeters located along the detection lines, measures the positions and orientations of the optical modules with an accuracy of about $10 \mathrm{~cm}$. A detailed description of the detector is given in ref. [11].

The arrival time and charge of the photo-multiplier tube signals are digitised [12] and 
transmitted to shore. The absolute time stamping is performed by interfacing the clock system to the GPS which provides a time accuracy of about $100 \mathrm{~ns}$ with respect to Universal Time Coordinated [13]. The data are dominated by optical background due to bioluminescence and natural radioactive decays. On shore, the physics signals are selected from the data stream by a software data filter that operates in real time. This data filter has multiple algorithms implemented, each designed to find a specific physics signal. The physics events are stored on disk for offline reconstruction. A detailed description of the data acquisition and data filtering is given in ref. [14].

\section{Data selection}

The analysis described in this paper has been applied to data collected in 2007, in the period from January 27 to December 7 . At this time, the ANTARES detector was still under construction and consisted of 5 detection lines.

\subsection{GRB selection}

The GRBs examined for neutrino emission were selected from the observations of GRBs by satellite instruments, as archived by the Gamma-ray Burst Coordinates Network (GCN) [15, 16]. In order to suppress the background from muons produced by cosmic ray interactions in the atmosphere above the detector, hereafter referred to as atmospheric muons, GRBs were selected that occurred below the horizon of the ANTARES detector. Neutrinos from such GRBs would traverse the Earth and cross the detector in an upward-going direction. In the period considered, 46 GRBs occurred below the ANTARES horizon during physics data taking.

By requiring the availability of detector alignment data, and by applying quality criteria based on the environmental conditions, the data in coincidence with 6 GRBs were excluded. The time and position information of the 40 remaining GRBs, listed in table 1 , were used to search for a correlated neutrino signal. A total of 32 GRBs were detected by the Swift satellite [17], 4 by INTEGRAL [18] and 4 by other satellites of the Third Interplanetary Network [19].

\subsection{Data processing}

This analysis focuses on the search for (anti)muons, produced by muon-(anti)neutrino charged current interactions. Throughout the rest of this paper, muon-neutrinos denotes both muonneutrinos and muon-antineutrinos. A data filter algorithm that is sensitive to muons from any direction has been applied during data taking. This algorithm first selects photons detected within 20 ns by separate optical modules in the same triplet. It then identifies a muon by requiring that for at least 5 triplets the relative arrival times of these photons are compatible with the signal expected from a muon traversing the detector. The muon purity for this data filter algorithm is better than $90 \%$. Its average event rate was $1.0 \mathrm{~Hz}$ in the period considered and is mainly due to the background from atmospheric muons.

These events were reconstructed offline to determine the muon trajectory, using a multistage fitting procedure. The reconstruction code follows the algorithm described in ref. [20]. Minor modifications were made to improve agreement between data and Monte Carlo. The most important modification compared to ref. [20] is that the amplitude information of the detected photons (hereafter referred to as hits) is discarded. As a result, the initial selection of signal hits is purely based on coincidences and the causality criterion. The algorithm 


\begin{tabular}{|c|c|c|c|c|c|}
\hline GRB & GCN Circular & GCN Report & GRB & GCN Circular & GCN Report \\
\hline GRB070207 & 6089 & & GRB070724 & & 74.2 \\
\hline GRB070209 & 6101 & 32.2 & GRB070729 & & 77.1 \\
\hline GRB070227 & & 37.1 & GRB070805 & 6711 & \\
\hline GRB070311 & 6189 & & GRB070808 & 6724 & \\
\hline GRB070326 & 6653 & & GRB070810B & & 81.1 \\
\hline GRB070328 & & 42.3 & GRB070821 & 6766 & \\
\hline GRB070330 & & 43.2 & GRB070824 & 6768 & \\
\hline GRB070419 & & 47.1 & GRB070911 & 6810 & 82.2 \\
\hline GRB070419B & & 49.1 & GRB070913 & & 88.1 \\
\hline GRB070420 & & 48.1 & GRB070923 & 6821 & \\
\hline GRB070429B & 7140 & 51.1 & GRB071001 & & 86.1 \\
\hline GRB070508 & & 54.2 & GRB071003 & & 87.2 \\
\hline GRB070509 & & 55.1 & GRB071008 & & 91.1 \\
\hline GRB070517 & & 56.2 & GRB071010 & 6864 & 89.2 \\
\hline GRB070611 & & 63.3 & GRB071010B & 6888 & 92.1 \\
\hline GRB070612 & 6556 & 64.1 & GRB071017 & 6927 & \\
\hline GRB070612B & & 65.1 & GRB071028B & & 105.1 \\
\hline GRB070615 & 6537 & & GRB071031 & & 99.1 \\
\hline GRB070707 & 6615 & & GRB071112B & & 103.1 \\
\hline GRB070721 & & 72.2 & GRB071117 & & 106.2 \\
\hline
\end{tabular}

Table 1. List of the GRBs that are included in the analysis. The column "GRB" indicates the name of the GRB. The columns "GCN Circular" and "GCN Report" indicate the numbers of the GCN Circulars archive [15] and GCN Reports [16] respectively, from which the measured GRB data were taken.

starts with a linear prefit which is used as a starting track for the subsequent stages. In addition, eight different starting tracks are generated by rotating and translating the result of the prefit. This is to increase the probability to find the global maximum of the likelihood function. The final stage of the fitting procedure consists of a maximum likelihood fit of the measured photon arrival times. A quality parameter for the fit, indicated by $\Lambda$, is determined based on the final value of the likelihood function. The $\Lambda$ parameter is quantified by

$$
\Lambda \equiv \frac{\log (L)}{N_{\text {hits }}-5}+0.1 \times\left(N_{\text {comp }}-1\right),
$$

which incorporates the maximum value of the likelihood, $L$, and the number of degrees of freedom of the fit, i.e. the number of hits, $N_{\text {hits }}$, used in the fit minus the number of fit parameters; $N_{\text {comp }}$ is the number of times the repeated initial steps of the reconstruction converged to the same result. In general, $N_{\text {comp }}=1$ for badly reconstructed events while it can be as large as nine for well reconstructed events. The coefficient $0.1 \mathrm{in} \mathrm{eq.} 3.1$ was chosen to maximise the separation in $\Lambda$ between simulated signal and misreconstructed downgoing muons. The same reconstruction algorithm was used for the analysis described in ref. [21] which contains a brief description of the algorithm. For a more detailed description of the reconstruction algorithm see ref. [20].

The fit also provides an estimate of the uncertainties on the track parameters. These are used to select events with a well defined direction. The distribution of the estimated angular uncertainty on the direction of the reconstructed muon track, indicated by $\beta$, is shown in 

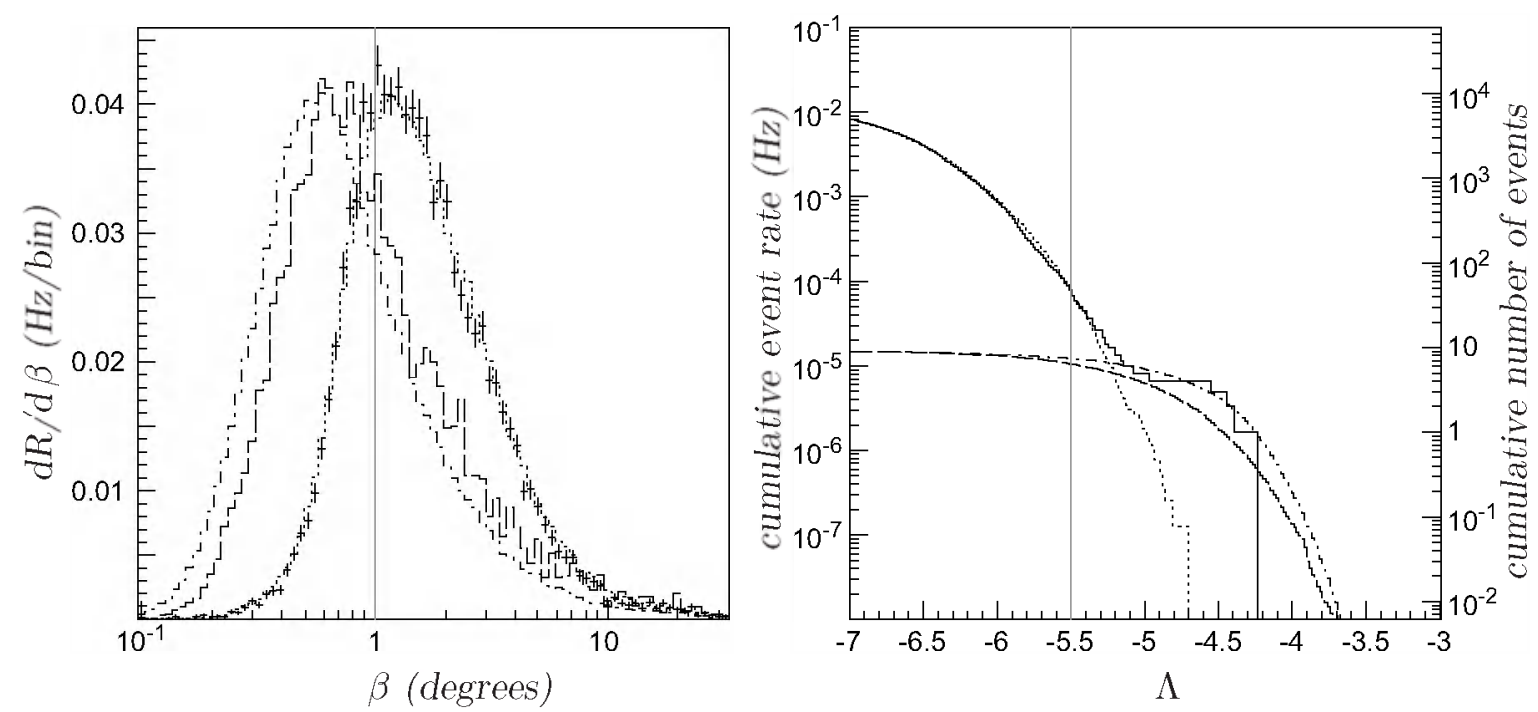

Figure 2. Left: Distribution of the estimated angular uncertainty $\beta$ on the direction of the reconstructed muon track for upward reconstructed simulated atmospheric muons (dotted line), atmospheric muon-neutrinos $\times 1300$ (dashed line) and signal events with an $E^{-2}$ spectrum (dash-dotted line) normalised to the atmospheric muon-neutrinos, compared to upward reconstructed events in the data covering about 7 days and containing the 40 selected GRBs (data points), without further cuts. Right: Cumulative $\Lambda$ distribution for upward reconstructed simulated atmospheric muons (dotted line), atmospheric muon-neutrinos (dashed line) and signal events with an $E^{-2}$ spectrum (dash-dotted line) normalised to the atmospheric muon-neutrinos, compared to upward reconstructed events in the data covering about 7 days and containing the 40 selected GRBs (solid line), for events with $\beta \leq 1^{\circ}$. The vertical lines indicate the analysis cuts.

figure 2 (left). Since all selected GRBs occurred below the ANTARES horizon, only events with an upward reconstructed direction are considered.

Due to the changing detector conditions during the period considered, and the varying environmental conditions, the statistics of the uncorrelated data that are equivalent to the conditions during the GRBs is too limited to estimate the background. Hence the background estimate has been made based on simulations. Figure 2 includes the expected distributions from atmospheric muons, atmospheric muon-neutrinos as well as an assumed signal with an $E^{-2}$ spectrum, obtained from simulations. The neutrino signal is generated with software packages [22] that simulate the neutrino interaction as well as the production and propagation of charged particles. The simulation uses the model for the atmospheric muon-neutrino flux from ref. [23]. The atmospheric muon contribution is simulated with the MUPAGE package [24], which is based on a full Monte Carlo simulation of primary cosmic ray interactions and shower propagation in the atmosphere and reproduces the MACRO data $[25,26]$. The simulated atmospheric muon contribution has an equivalent live time of one month.

In the simulations, the stochastic energy loss of the muons, the production and propagation of the Cherenkov photons, the response of the photo-multiplier tubes to Cherenkov light and the simulation of the detector electronics are all included. The simulated photon signals are processed with the same data filter and reconstruction algorithms as the data. To obtain a realistic simulation of the varying environmental conditions due to bioluminescence, the measured optical background in coincidence with the prompt emission of each of the selected GRBs is taken from the data and added to the simulated events. 

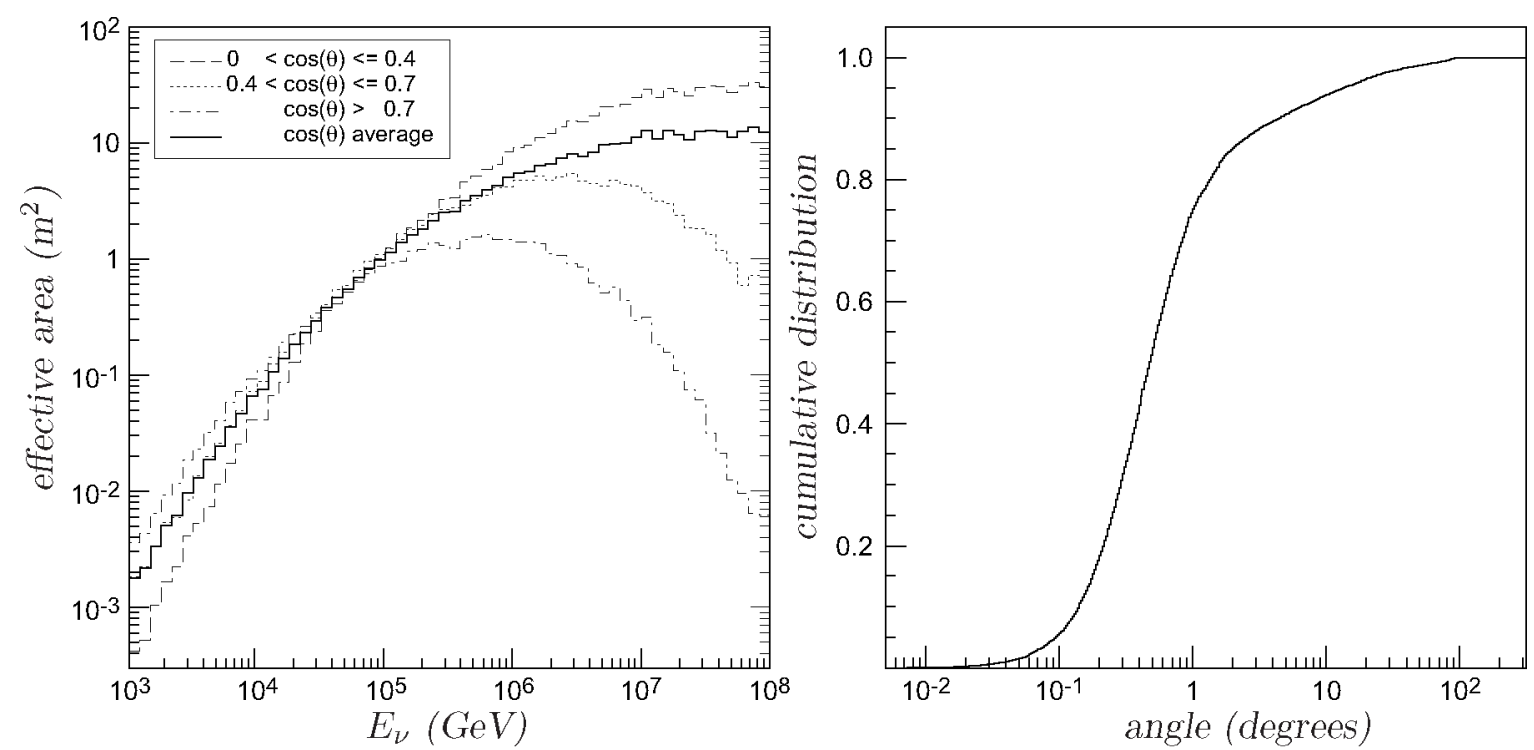

Figure 3. Left: the angle averaged neutrino effective area of the detector consisting of 5 detection lines, and the effective areas for different neutrino zenith angle bins $(\cos (\theta)=1$ corresponds to vertically upward). The selection cuts are included. Right: the cumulative distribution of the angle between the reconstructed muon direction and the true neutrino direction for the detector consisting of 5 detection lines. The distribution is shown for selected events with an $E_{\nu}^{-2}$ spectrum.

Selection cuts on the $\Lambda$ and $\beta$ parameters are set to achieve a reliable rejection of misreconstructed atmospheric muons, while keeping a high signal efficiency. Neutrino candidates are required to be reconstructed as upgoing muons, with an estimated angular uncertainty $\beta \leq 1^{\circ}$, see figure 2 (left). This cut removes $72 \%$ of the misreconstructed atmospheric muons. Figure 2 (right) shows the cumulative $\Lambda$ distribution for upgoing reconstructed events, where the cut of $\beta \leq 1^{\circ}$ has been applied. Neutrino candidates are, in addition, required to have a quality value of $\Lambda \geq-5.5$. The cuts on the $\Lambda$ and $\beta$ parameters are chosen such that the background rate is reduced to a level below $2 \times 10^{-5} \mathrm{~Hz} \mathrm{sr}^{-1}$, while the signal efficiency is about $60 \%$ for the models considered in this analysis. The strong constraints from the time and direction coincidence with a GRB allow for a looser cut on the quality parameter $\Lambda$ than that applied for the ANTARES point source search [27].

In both plots of figure 2 , the contribution from atmospheric muons was scaled by 0.85 to reproduce the data. This scaling factor is well within the uncertainty on the flux normalisation $[28]$.

The neutrino effective area of the detector consisting of 5 detection lines is shown in figure 3 (left) as a function of the neutrino energy. The presented effective areas include the selection cuts and are the average of the effective areas for muon-neutrinos and muonantineutrinos. The angle averaged effective area is shown as well as the effective areas for different neutrino zenith angle bins. For vertically upward-going neutrinos the effective area is suppressed at high energies due to the absorption in the Earth.

The angular resolution of the detector is determined by the angular uncertainty on the reconstructed muon direction $(\beta)$ and the neutrino scattering angle. The distribution of the angle between the reconstructed muon direction and the true neutrino direction for the detector consisting of 5 detection lines was evaluated with simulations. The cumulative 

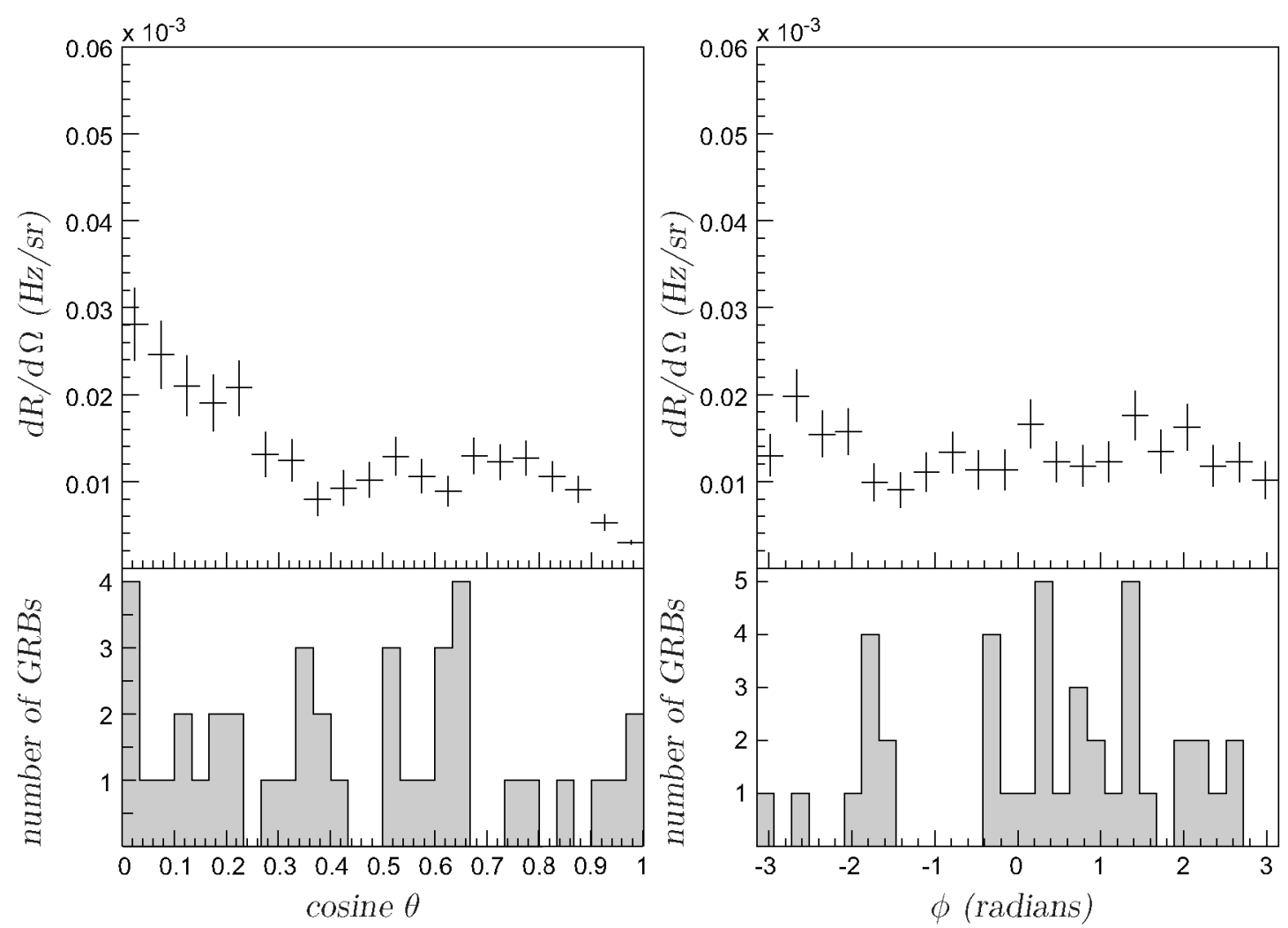

Figure 4. Top: the estimated background rate per unit solid angle as a function of the reconstructed muon direction in detector coordinates (left: zenith angle cosine $\theta$, right: azimuthal angle $\phi$ in radians; $\cos (\theta)=1$ corresponds to vertically upward). Bottom: the location of each of the 40 selected GRBs.

distribution of this angle is shown in figure 3 (right) for events with a neutrino spectrum proportional to $E_{\nu}^{-2}$ that passed the selection cuts. The median of this angular resolution was estimated to be $0.5 \pm 0.1$ degrees.

\section{Data analysis}

The remaining background due to atmospheric muon-neutrinos (89\%) and misreconstructed atmospheric muons (11\%), after the cuts on $\beta$ and $\Lambda$, was estimated from simulations. The measured optical background from the data in coincidence with the prompt emission of each of the selected GRBs was added to the simulated events. The background rate per unit solid angle as a function of the reconstructed muon direction in detector coordinates is shown in figure 4 (top plots). The contribution from atmospheric muons was scaled by 0.85 to reproduce the data. The background rate as a function of the reconstructed zenith angle, $\theta$, is less isotropic compared to the background rate as a function of the reconstructed azimuthal angle, $\phi$. However, since the selected GRBs are distributed rather isotropically (bottom plots of figure 4), the average background rate may be used for each GRB. The expected background rate per unit solid angle is estimated by taking a weighted average of the background rate in each solid angle bin, where the weighting accounts for the relative duration of GRBs in that solid angle bin. This results in an estimated background rate of $1.54 \times 10^{-5} \mathrm{~Hz} \mathrm{sr}^{-1}$. 
A neutrino candidate is considered to come from the GRB when the reconstructed muon track points back to the GRB within $2^{\circ}$. From simulations it was estimated that for about $85 \%$ of the neutrino candidates, the muon is reconstructed within $2^{\circ}$ from the neutrino direction. This high signal efficiency is the result of the selection of events with a good angular resolution (the cut on the $\beta$ parameter). The uncertainty on the source positions of the GRBs considered (table 1) is smaller than $0.07^{\circ}$. The total background, $\mu_{b k g}$, due to atmospheric muon-neutrinos and misreconstructed atmospheric muons during the prompt emission of the 40 GRBs can be expressed as

$$
\mu_{b k g}=\frac{d R}{d \Omega} \times \Omega \times T
$$

where $\frac{d R}{d \Omega}$ is the background rate per unit solid angle, $\Omega$ is the solid angle of the $2^{\circ}$ search cone and $T$ is the total prompt emission duration of the 40 GRBs. For the prompt emission duration of the GRB, the so called $T_{90}$ time interval is taken [29], extended by $5 \%$ before the start, and $5 \%$ after the end of this time interval. $T$ amounts to 2114 seconds. The total estimated background amounts to $1.24 \times 10^{-4}$ events.

\section{Results}

This analysis focuses only on the detection of muon-neutrinos in coincidence with the prompt emission phase of the GRB. The search for neutrinos in correlation with GRBs was done in a stacking analysis, in which all data in coincidence with the prompt emission of the GRBs were accumulated.

Selected neutrino candidates are considered to be correlated with a GRB when their detection time is in coincidence with the prompt emission of the GRB, assuming that neutrinos travel at the speed of light. After unblinding the data, no neutrino candidates were found in correlation with the selected GRBs.

\subsection{GRB neutrino spectra}

A general neutrino spectrum of $E_{\nu}^{-2}$ is assumed for the neutrino emission from GRBs, where $E_{\nu}$ is the neutrino energy. In addition, three other energy spectra for neutrino emission from GRBs have been considered: the energy spectrum proposed by Guetta et al. [30], the general Waxman and Bahcall energy spectrum [31], and the energy spectrum proposed by Ahlers et al. [32].

The energy spectrum according to Guetta et al. is calculated for each GRB individually using the data from the instruments on the satellite that detected the GRB, taken from the references given in table 1 . The method to calculate a neutrino fluence from an individual GRB is provided in ref. [30]. The prediction therein referred to as "Model 2" is used. In case not all required parameters were measured, nor default values are provided in ref. [30], the values as listed in table 2 were used. Within the list of GRBs no single GRB yields a detectable signal. The total estimated muon-neutrino fluence for the 40 selected GRBs according to Guetta et al. is the sum of the calculated individual muon-neutrino fluences, and is shown in figure 5 (left). The total number of expected events from the 40 selected GRBs for the estimated muon-neutrino fluence with the energy spectrum according to Guetta et al. is $1.7 \times 10^{-3}$. The estimated muon-neutrino fluence represents the fluence at Earth and includes the effect of neutrino oscillations.

Waxman and Bahcall [31] provide an approximate estimate of the neutrino energy spectrum, which is assumed to be the same for each GRB. The expected number of events for 40 


\begin{tabular}{ll}
\hline Parameter & Value \\
\hline$z$ & 0.25 or 2.8 \\
$\epsilon_{e}$ & 0.1 \\
$\epsilon_{B}$ & 0.1 \\
$\epsilon_{\gamma}^{b}$ & $0.4 \mathrm{MeV}$ \\
$\alpha_{\gamma}$ & 1 \\
$\beta_{\gamma}$ & 2 \\
$L_{\gamma}$ & $10^{51} \mathrm{ergs} \mathrm{s}^{-1}$ \\
$F_{\gamma}$ & $6 \times 10^{-6} \mathrm{ergs} \mathrm{c}^{-2}$ \\
\hline
\end{tabular}

Table 2. Assumed parameter values used for calculating the neutrino spectrum for an individual GRB using the method described in ref. [30] (see text). The symbols correspond to those used in ref. [30]. The redshift $z$ is set to 0.25 for GRBs with a prompt emission duration of less than $2 \mathrm{~s}$ [33], and set to 2.8 for other GRBs [34]; the values for the fraction of the internal energy in electrons $\left(\epsilon_{e}\right)$ and the magnetic field $\left(\epsilon_{B}\right)$ are taken from [35]; the default values for the break energy in the $\gamma$-ray spectrum $\varepsilon_{\gamma}^{b}$, the spectral indices of the $\gamma$-ray spectrum before $\left(\alpha_{\gamma}\right)$ and after $\left(\beta_{\gamma}\right) \varepsilon_{\gamma}^{b}$, the $\gamma$-ray luminosity $L_{\gamma}$, and the $\gamma$-ray fluence $F_{\gamma}$ are the mean values from the Swift catalogue [36, 37].
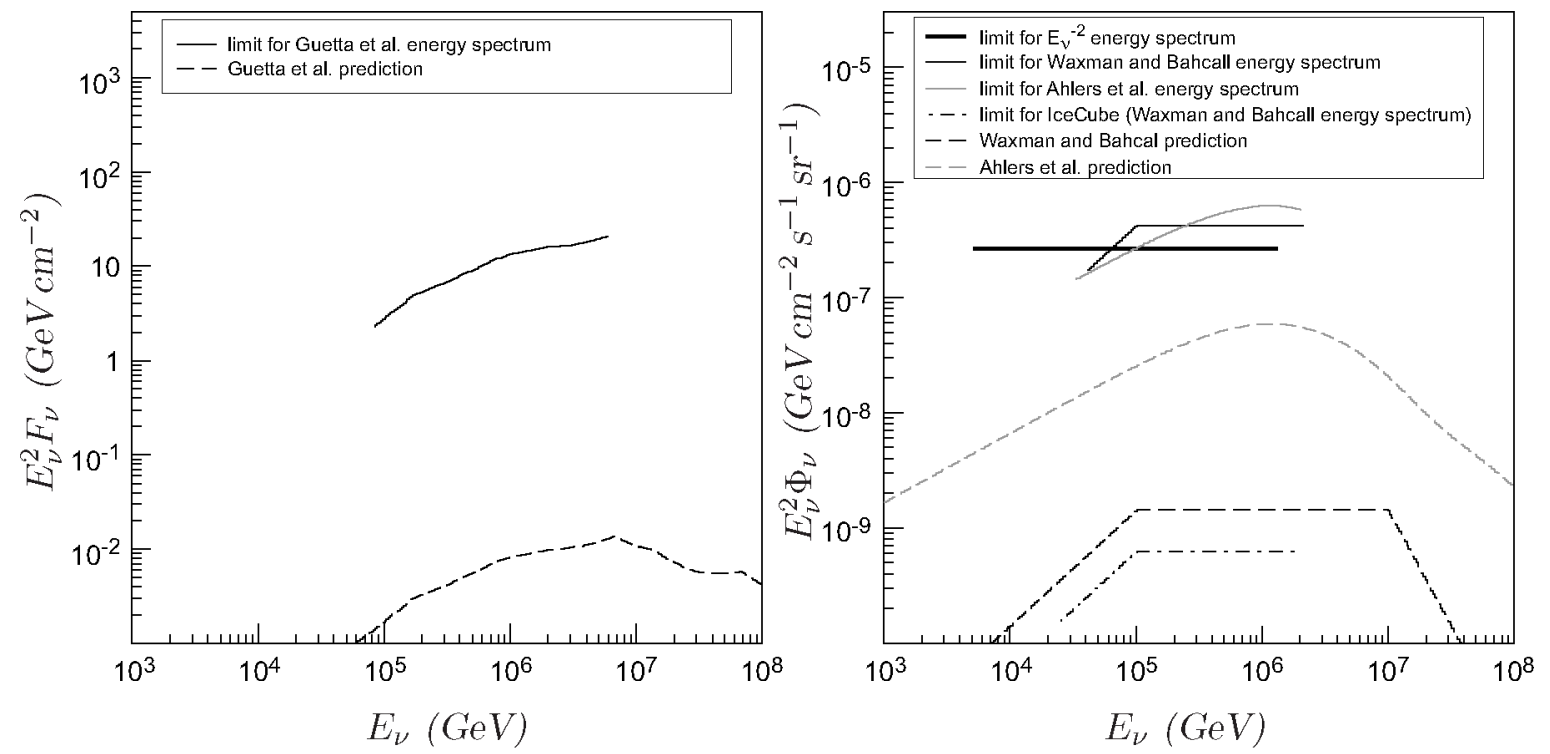

Figure 5. Left: the $90 \% C L$ upper limit on the muon-neutrino fluence $F_{\nu}$ from the 40 selected GRBs for the energy spectrum according to Guetta et al. (solid line) and the corresponding total estimated muon-neutrino fluence including oscillations (dashed line). Right: the 90\% CL upper limit on the diffuse muon-neutrino flux $\Phi_{\nu}$ for the $E_{\nu}^{-2}$ energy spectrum (thick solid line), the Waxman and Bahcall energy spectrum (thin black line), and the energy spectrum according to Ahlers et al. (thin grey line). The black dash-dotted line is the 90\% CL upper limit of IceCube [2] assuming the Waxman and Bahcall energy spectrum. Also shown are the estimated diffuse muon-neutrino fluxes including oscillations assuming the Waxman and Bahcall energy spectrum (black dashed line) and that according to Ahlers et al. (grey dashed line).

GRBs assuming the Waxmann and Bahcall energy spectrum is $7.0 \times 10^{-3}$. The estimated GRB muon-neutrino intensity assuming a Waxman and Bahcall energy spectrum is presented as a diffuse flux in figure 5 (right) where a total of $10^{3}$ GRBs are expected per year. ${ }^{1}$ The

\footnotetext{
${ }^{1}$ In ref. [31] the evolution correction is assumed to be 1.
} 
right plot in figure 5 also includes an estimated diffuse muon-neutrino flux according to Ahlers et al. [32] where typical values for the parameters of the GRB environment were used as provided in ref. [32]. The estimated diffuse muon-neutrino fluxes for both energy spectra represent the fluxes at Earth and include the effect of neutrino oscillations.

\subsection{Upper limits on the diffuse neutrino flux and the neutrino fluence}

Since no events were found in correlation with the prompt photon emission of the 40 selected GRBs, upper limits have been placed on the intensity of the diffuse muon-neutrino flux and the muon-neutrino fluence at Earth for the different models. The $90 \%$ confidence level limits were set using the Feldman-Cousins prescription [38], and are shown in figure 5. The same systematic uncertainties as described in ref. [27] have been considered. These include the effect of reduced optical module efficiencies and the effects which have a net result of degrading the time resolution, such as possible mis-alignments of the detector, inaccuracies in the simulation of light propagation in the water or in the transit time distribution of the photo-multiplier tubes. The impact of the systematic uncertainties were evaluated by including these effects in the simulation, described in ref. [27]. This results in a degradation of the limits of less than $10 \%$.

For the $E_{\nu}^{-2}$ energy spectrum, $90 \%$ of the signal is expected in the energy range $5.2 \mathrm{TeV}<E_{\nu}<1.4 \mathrm{PeV}$. The upper limit on the diffuse muon-neutrino flux for the $E_{\nu}^{-2}$ energy spectrum is $2.7 \times 10^{-7}\left(E_{\nu} / \mathrm{GeV}\right)^{-2} \mathrm{GeV}^{-1} \mathrm{~cm}^{-2} \mathrm{~s}^{-1} \mathrm{sr}^{-1}$. For the energy spectrum according to Waxman and Bahcall $90 \%$ of the signal is expected in the energy range $41 \mathrm{TeV}<E_{\nu}<2.1 \mathrm{PeV}$ and a 90\% CL upper limit of about 294 times the predicted flux as shown in figure 5 (right) was set. For the energy spectrum according to Ahlers et al. $90 \%$ of the signal is expected in the energy range $33 \mathrm{TeV}<E_{\nu}<2.0 \mathrm{PeV}$ and a 90\% CL upper limit of about 11 times the predicted flux as shown in figure 5 (right) was set. For the energy spectrum according to Guetta et al. $90 \%$ of the signal is expected in the energy range $86 \mathrm{TeV}<E_{\nu}<6.0 \mathrm{PeV}$ and a $90 \% \mathrm{CL}$ upper limit of about 1467 times the predicted fluence as shown in figure 5 (left) was set.

\section{Conclusions}

A search for muon-neutrinos in correlation with the prompt emission of gamma-ray bursts using the data taken with the ANTARES detector during the first year of operation has been presented. During the period considered, the detector was less than half its final size. The 40 GRBs that were examined for neutrino emission were selected from the GRB observations by satellite instruments. No correlations between neutrinos and the selected GRBs have been found. Upper limits have been obtained on the fluence of muon-neutrinos from the 40 GRBs and on the diffuse muon-neutrino flux for different models.

A neutrino telescope in the Mediterranean Sea is well suited to detect high-energy neutrinos in correlation with GRBs spread over a wide region of the sky, including in particular the Southern hemisphere. The low background is the result of the short duration of GRBs and the excellent angular resolution. The ANTARES detector was completed mid 2008 and is 2.5 times bigger than the detector configuration considered in this analysis. Since the completion of the detector, on average 250 GRBs per year have been detected in the Southern hemisphere and at least five more years of data taking are foreseen. With this, a large sample of GRBs is available for further analysis, complementary to the IceCube field of view and energies. The next-generation neutrino telescope KM3NeT [9], to be built in the Mediterranean 
Sea, will surpass the ANTARES sensitivity by two orders of magnitude. Once operational it will probe GRB models with unprecedented sensitivity, and will push the boundaries towards new discoveries.

\section{Acknowledgments}

The authors acknowledge the financial support of the funding agencies: Centre National de la Recherche Scientifique (CNRS), Commissariat á l'énergie atomique et aux énergies alternatives (CEA), Agence National de la Recherche (ANR), Commission Européenne (FEDER fund and Marie Curie Program), Région Alsace (contrat CPER), Région Provence-Alpes-Côte d'Azur, Département du Var and Ville de La Seyne-sur-Mer, France; Bundesministerium für Bildung und Forschung (BMBF), Germany; Istituto Nazionale di Fisica Nucleare (INFN), Italy; Stichting voor Fundamenteel Onderzoek der Materie (FOM), Nederlandse organisatie voor Wetenschappelijk Onderzoek (NWO), the Netherlands; Council of the President of the Russian Federation for young scientists and leading scientific schools supporting grants, Russia; National Authority for Scientific Research (ANCS - UEFISCDI), Romania; Ministerio de Ciencia e Innovación (MICINN), Prometeo of Generalitat Valenciana and MultiDark, Spain; Agence de l'Oriental, Morocco. We also acknowledge the technical support of Ifremer, AIM and Foselev Marine for the sea operation and the CC-IN2P3 for the computing facilities.

\section{References}

[1] P. Mészáros, Gamma-ray bursts, Rep. Prog. Phys. 69 (2006) 2259

[2] R. Abbasi et al., An absence of neutrinos associated with cosmic-ray acceleration in $\gamma$-ray bursts, Nature 484 (2012) 351

[3] A. Achterberg et al., The Search for Muon Neutrinos from Northern Hemisphere Gamma-Ray Bursts with AMANDA, Astrophys. J. 674 (2008) 357

[4] R. Abbasi et al., Search for Muon Neutrinos from Gamma-ray Bursts with the IceCube Neutrino Telescope, Astrophys. J. 710 (2010) 346

[5] R. Abbasi et al., Limits on Neutrino Emission from Gamma-Ray Bursts with the 40 String IceCube Detector, Phys. Rev. Lett. 106 (2011) 141101

[6] A.G. Vieregg et al., The First Limits on the Ultra-high Energy Neutrino Fluence from Gamma-ray Bursts, arXiv:1102.3206

[7] S. Fukuda et al., Search for Neutrinos from Gamma-Ray Bursts Using Super-Kamiokande, Astrophys. J. 578 (2002) 317

[8] A.V. Avrorin et al., Search for neutrinos from gamma-ray bursts with the Baikal neutrino telescope NT200, Astron. Lett. 37 (2011) 692

[9] KM3NeT Technical Design Report, ISBN 978-90-6488-033-9

[10] P. Amram et al., The ANTARES optical module, Nucl. Instrum. Meth. A 484 (2002) 369

[11] J.A. Aguilar et al., ANTARES: The first undersea neutrino telescope, Nucl. Instrum. Meth. A 656 (2011) 11

[12] J.A. Aguilar et al., Performance of the front-end electronics of the ANTARES neutrino telescope, Nucl. Instrum. Meth. A 622 (2010) 59

[13] J.A. Aguilar et al., Time calibration of the ANTARES neutrino telescope, Astropart. Phys. 34 (2011) 539 
[14] J.A. Aguilar et al., The data acquisition system for the ANTARES neutrino telescope, Nucl. Instrum. Meth. A 570 (2007) 107

[15] http://gcn.gsfc.nasa.gov/gen3_archive.html

[16] http://gcn.gsfc.nasa.gov/report_archive.html

[17] N. Gehrels et al., The Swift Gamma-Ray Burst Mission, Astrophys. J. 611 (2004) 1005

[18] S. Mereghetti et al., The Integral Burst Alert System (IBAS), Astron. Astrophys. 411 (2003) L291

[19] K. Hurley et al., Mars Odyssey Joins the Third Interplanetary Network, Astrophys. J. Supp. S. 164 (2006) 124

[20] A. J. Heijboer, Track Reconstruction and Point Source Searches with ANTARES, PhD thesis (2004), http://www.nikhef.nl/pub/services/newbiblio/theses.php

[21] S. Adrián-Martínez et al., Search for cosmic neutrino point sources with four years of data from the ANTARES telescope, Astrophys. J. 760 (2012) 53

[22] D.J.L. Bailey, Monte Carlo tools and analysis methods for understanding the ANTARES experiment and predicting its sensitivity to Dark Matter, PhD thesis (2002), http://antares.in2p3.fr/Publications/index.html

[23] V. Agrawal et al., Atmospheric neutrino flux above 1 GeV, Phys. Rev. D 53 (1996) 1314

[24] G. Carminati et al., Atmospheric MUons from PArametric formulas: a fast GEnerator for neutrino telescopes (MUPAGE), Comput. Phys. Commun. 179 (2008) 915

[25] M. Ambrosio et al., High energy cosmic ray physics with underground muons in MACRO. I. Analysis methods and experimental results, Phys. Rev. D 56 (1997) 1407; High energy cosmic ray physics with underground muons in MACRO. II. Primary spectra and composition, Phys. Rev. D 56 (1997) 1418

[26] M. Ambrosio et al., High statistics measurement of the underground muon pair separation at Gran Sasso, Phys. Rev. D 60 (1999) 032001

[27] S. Adrián-Martínez et al., First Search for Point Sources of High-Energy Cosmic Neutrinos with the ANTARES Neutrino Telescope, Astrophys. J. Lett. 743 (2011) L14

[28] J. A. Aguilar et al., Zenith distribution and flux of atmospheric muons measured with the 5-line ANTARES detector, Astropart. Phys., 34 (2010) 179

[29] C. Kouveliotou et al., Identification of Two Classes of Gamma-Ray Bursts, Astrophys. J. 413 (1993) L101

[30] D. Guetta et al., Neutrinos from individual gamma-ray bursts in the BATSE catalog, Astropart. Phys. 20 (2004) 429

[31] E. Waxman and J. Bahcall, High energy neutrinos from astrophysical sources: An upper bound, Phys. Rev. D 59 (1998) 023002

[32] M. Ahlers et al., GRBs on probation: Testing the UHE CR paradigm with IceCube, Astropart. Phys. 35 (2011) 87

[33] C. Hopman et al., The Redshift Distribution of Short Gamma-Ray Bursts from Dynamically Formed Neutron Star Binaries, Astrophys. J. Lett. 643 (2006) L91

[34] P. Jakobsson et al., A mean redshift of 2.8 for Swift gamma-ray bursts, Astron. Astrophys. 447 (2006) 897

[35] E. Waxman et al., Implications of the Radio Afterglow from the Gamma-Ray Burst of 1997 May 8, Astrophys. J. 497 (1998) 288 
[36] N.R. Butler et al., A Complete Catalog of Swift Gamma-Ray Burst Spectra and Durations: Demise of a Physical Origin for Pre-Swift High-Energy Correlations, Astrophys. J. 671 (2007) 656

[37] N.R. Butler et al., The Cosmic Rate, Luminosity Function, and Intrinsic Correlations of Long Gamma-Ray Bursts, Astrophys. J. 711 (2010) 495

[38] G.J. Feldman and R.D. Cousins, Unified approach to the classical statistical analysis of small signals, Phys. Rev. D 57 (1998) 3873 